\title{
Absolute judgments of simultaneously presented visual and auditory stimuli'
}

\author{
Peter H. Lindsay, Lola L. Cuddy and Endel Tulving \\ UNIVERSITY OF TORONTO
}

\begin{abstract}
Abstraet
Four Ss made judgments about unidimensional visual and auditory stimuli. Stimuli were judged under two levels of stimulus duration $-50 \mathrm{msec}$. or $2 \mathrm{sec}$., and two conditions of presentation-stimuli presented singly from one modality or simultaneously from both modalities. For both stimulus duration, the amount of transmitted information per modality was less under conditions of simultaneous presentation. Results were discussed in relation to the problem of divisibility of attention.
\end{abstract}

\section{Problem}

One type of experiment to study the problem of divisibility of attention requires $\mathrm{Ss}$ to perform on a task involving simultaneous presentation of information from two sensory modalities. Experiments in which different verbal messages are presented at the same time visually and aurally (e.g., Mowbray, 1952, 1954) have shown that simultaneous messages cannot be handled as efficiently as the same messages presented one at a time.

The present experiment was concerned with processing of simultaneously presented visual and auditory information in the absolute judgments task (Garner, 1962). This task allows for the exact control of duration of stimuli, it minimizes the importance of memory factors, and it makes it possible to use a performance measure-amount of information transmitted-which is equally applicable to both modalities. The problem was to determine whether perceptual identification of simple stimuli varying in only one dimension is affected by the requirement that $S$ attend to a stimulus from another modality presented at the same time.

\section{Method}

The visual stimuli were circles of white light on a dark background, while auditory stimuli consisted of $1000 \mathrm{cps}$ pure tones. Both stimuli varied along the dimension of intensity. Eight levels of intensity, separated by $.3 \mathrm{log}$ unit steps, were used in each modality. The S's task was to assign a numerical value from 1 to 8 to each stimulus presented, the assigned value depending upon the stimulus intensity.

A stimulus from a given modality was presented either alone or simultaneously with a stimulus from another modality. In Task $\mathrm{V}$ visual stimuli, and in Task A auditory stimuli were presented and judged alone. In Task V-A a visual stimulus and an auditory stimulus were presented simultaneously, and $S$ was told to "identify both stimuli, but concentrate on and report the visual stimulus first, and the auditory stimulus second." In Task A-V two stimuli were also presented simultaneously, but $\mathrm{S}$ was instructed to "concentrate on and report the auditory stimulus first, and the visual stimulus second." Thus, stimuli from a given modality, either visual or auditory, were judged under three different conditions; (1) single presentation of stimuli, (2) simultaneous presentation, the given modality reported first, and (3) simultaneous presentation, the given modality reported second. Two further independent variables were included; (1) duration of the stimulus, $50 \mathrm{msec}$. or 2 sec., and (2) presence or absence of feedback. Under the conditions of simultaneous presentation, the stimuli from two modalities were always of the same duration. Under feedback conditions Ss were informed of the correct response after they had made their own identifying response for a single stimulus or a pair of stimuli.

Each of four Ss, undergraduate students paid for their services, served under all conditions of the experiment. The conditions were presented in a randomly determined order. Each condition was tested in a separate experimental session of approximately an hour's duration. On the day before the first experimental session, each $\mathrm{S}$ was given a practice session involving both single and simultaneous stimuli of both $50 \mathrm{msec}$, and $2 \mathrm{sec}$. duration.

In a given experimental session, either 256 single stimuli or 256 simultaneous pairs of stimuli were presented, each of the eight stimulus intensities in a given modality occurring 32 times in a random sequence. Stimuli were presented at intervals of $12 \mathrm{sec}$. A warning signal preceded the presentation of each stimulus.

\section{Results}

For each $\mathrm{S}$ the amount of information transmitted in bits (T) was computed on the basis of 256 responses for a given modality in an experimental session. The raw data thus consisted of $96 \mathrm{~T}$ scores: 4 Ss $\times 2$ modalities $\mathrm{x} 3$ tasks $\times 2$ durations $\times 2$ conditions of feedback. Table 1 shows the mean $T$ scores for the four Ss under various combinations of experimental treatments.

Analysis of variance showed that all main effects were significant at better than the .001 level. Transmitted information under single stimulus conditions was greater than under simultaneous conditions for both modalities, and greater when a given modality was reported first than when it was reported second. Transmitted information for stimuli of 2 sec. duration was 
Table 1

Mean $\mathrm{T}$ (in bits) as a function of stimulus modality, task conditions, feedback conditions, and stimulus duration.

\begin{tabular}{|c|c|c|c|c|c|c|c|c|c|c|c|c|}
\hline \multirow{4}{*}{ Duration } & \multicolumn{6}{|c|}{ Visual Stimuli } & \multicolumn{6}{|c|}{ Auditory Stimuli } \\
\hline & \multirow{2}{*}{\multicolumn{2}{|c|}{$\begin{array}{l}\text { Single } \\
\text { stimuli }\end{array}$}} & \multicolumn{4}{|c|}{ Simultaneous stimuli } & \multirow{2}{*}{\multicolumn{2}{|c|}{$\begin{array}{l}\text { Single } \\
\text { stimuli }\end{array}$}} & \multicolumn{4}{|c|}{ Simultaneous stimuli } \\
\hline & & & \multicolumn{2}{|c|}{$\begin{array}{l}\text { Reported } \\
\text { first }\end{array}$} & \multicolumn{2}{|c|}{$\begin{array}{l}\text { Reported } \\
\text { second }\end{array}$} & & & \multicolumn{2}{|c|}{$\begin{array}{l}\text { Reported } \\
\text { first }\end{array}$} & \multicolumn{2}{|c|}{$\begin{array}{l}\text { Reported } \\
\text { second }\end{array}$} \\
\hline & F & $\mathrm{NF}$ & $\mathrm{F}$ & $\mathrm{NF}$ & $\mathrm{F}$ & $\mathrm{NF}$ & F & $\mathrm{NF}$ & F & $N F$ & F & $\mathrm{NF}$ \\
\hline $50 \mathrm{msec}$. & 1.64 & 1.58 & 1.53 & 1.33 & 1.32 & 1.15 & 1.39 & 1.34 & 1.46 & 1.16 & 1.21 & 1.08 \\
\hline $2 \mathrm{sec}$ & 2.00 & 1.61 & 1.70 & 1.58 & 1.42 & 1.42 & 1.65. & 1.53 & 1.61 & 1.41 & 1.45 & 1.44 \\
\hline
\end{tabular}

Note: F refers to feedback, NF to no feedback.

greater than for stimuli of $50 \mathrm{msec}$ duration. Over all conditions the two means were 1.57 and 1.35 bits, respectively. It also appeared that, by and large, reduction in information transmission over the three tasks was approximately the same for both durations. Finally, performance was greater under feedback conditions (mean $\mathrm{T}=1.53$ bits) than under non-feedback conditions (mean $T=1.39$ ), and greater for visual stimuli (mean $\mathrm{T}=1.52$ ) than for auditory stimuli (mean $\mathrm{T}=1.39$ ).

\section{Discussion}

The results show that a typical $\mathrm{S}$ cannot identify simple unidimensional stimuli as well under the conditions of simultaneous inputs from two modalities as he can when he has to deal with only one modality. The magnitude of this impairment is not very great, but it is statistically highly reliable. To the extent that such an impairment in the processing of simultaneously presented information from two different sources can be attributed to some sort of limitation in the mechanism of attention, these findings suggest that two attentive acts cannot be performed simultaneously without some loss in efficiency, even when the perceptual task appears to be rather simple. This finding corroborates similar findings from other experiments in which more complex stimulus materials and different response measures have been used (Mowbray, 1952, 1954). It is also reminiscent of the findings from experiments on absolute judgments involving two stimulus dimensions within the same modality, in which attending to two dimensions simultaneously leads to lower judgmental accuracy per dimension (Garner, 1962).
Two levels of stimulus duration were used in the experiment to assess the tenability of the hypothesis that Ss switch attention from one modality to another under the conditions of simultaneous presentation. Such switching, if it occurs, would be expected to be more feasible with $2 \mathrm{sec}$. stimuli than with $50 \mathrm{msec}$. stimuli. If switching facilitates identification of simultaneously presented stimuli, it would be expected that, relative to performance on single stimulus task, performance under the conditions of simultaneous presentation would be better for $2 \mathrm{sec}$. stimuli than for $50 \mathrm{msec}$. stimuli. No such effect was found. This leaves the problem of switching of attention unsettled. Either no switching occurred, or it occurred with approximately equal effects for both the long and short stimuli. Although the physical duration of the short stimuli was only $50 \mathrm{msec}$., information about stimuli may have been available to Ss for a longer period, in the form of perceptual tracés. The extended duration of the perceptual trace of the stimulus may have made switching possible.

Heferences

GARNER, W. R. Uncertainty and structure as psychological concepts. New York: Wiley, 1962.

MOWBRAY, G. H. Simultaneous vision and audition: The detection of elements missing from overlearned sequences. J. exp. Psychol., $1952,44,292-300$.

MOWBRAY, G. H. The perception of short phrases presented simultaneously for visual and auditory reception. Quart. J. exp. Psychol., 1954, 6, 86-92.

Note

1. This research was supported by the National Research Council of Canada, under Grant No. APT-39 to E. Tulving. 